\title{
Exponential convexity for majorization
}

\author{
Asif R Khan ${ }^{1,2^{*}}$, Naveed Latif ${ }^{2,3}$ and Josip Pečarić ${ }^{2,4}$
}

* Correspondence:

asif_rizkhan@yahoo.com

${ }^{1}$ Department of Mathematics,

University of Karachi, University Road, Karachi, Pakistan

Full list of author information is available at the end of the article

\begin{abstract}
In this article, we give more generalized results than in Anwar et al. (2010) and Latif and Pečarić (2010) in new direction by using second-order divided difference. We investigate the exponential convexity and logarithmic convexity for majorization type results by using class of continuous functions in linear functionals. We also construct positive semi-definite matrices for majorization type results. We will vary on choice of a family of functions in order to construct different examples of exponentially convex functions and construct some means. We also prove the monotonic property.

Mathematics Subject Classification (2000): 26A51; 39B62; 26D15; 26D20; 26 D99.
\end{abstract}

Keywords: convex function, majorization, second-order divided difference, positive semi-definite matrix, exponential-convexity, log-convexity

\section{Introduction and preliminaries}

Although inequalities play a fundamental role in nearly all branches of mathematics, inequalities are usually obtained by ad hoc methods rather than as consequences of some underlying "Theory of Inequalities". For certain kinds of inequalities, the notion of majorization leads to such a theory that is sometimes extremely useful and powerful for deriving inequalities. Moreover, the derivation of an inequality by methods of majorization is often very helpful both for providing a deeper understanding and for suggesting natural generalizations [1].

Now we define the notion of majorization as follows: For fixed $n \geq 2$, let

$$
\mathbf{x}=\left(x_{1}, \ldots, x_{n}\right), \quad \mathbf{y}=\left(y_{1}, \ldots, y_{n}\right)
$$

denote two $n$-tuples and

$$
x_{[1]} \geq x_{[2]} \geq \cdots \geq x_{[n]}, \quad y_{[1]} \geq y_{[2]} \geq \cdots \geq y_{[n]},
$$

be their ordered components.

Definition 1.1. [[1], p. 8] For $\mathbf{x}, \mathbf{y} \in \mathbb{R}^{n}$,

$$
\mathbf{x} \prec \mathbf{y} \text { if }\left\{\begin{array}{l}
\sum_{i=1}^{k} x_{[i]} \leq \sum_{i=1}^{k} y_{[i]}, \quad k=1, \ldots, n-1 ; \\
\sum_{i=1}^{n} x_{[i]}=\sum_{i=1}^{n} y_{[i]},
\end{array}\right.
$$

when $\mathbf{x} \prec \mathbf{y}, \mathbf{x}$ is said to be majorized by $\mathbf{y}(\mathbf{y}$ majorizes $\mathbf{x}$ ).

The following theorem is well-known in literature as the majorization theorem and a convenient reference for its proof is Marshall et al. [[1], p. 15, p. 157], which is due to Hardy et al. [[2], p. 75] and can also be found in [[3], p. 320]. For a discussion concerning the matter of priority see Mitrinović [[4], p. 169].

(c) 2012 Khan et al; licensee Springer. This is an Open Access article distributed under the terms of the Creative Commons Attribution License (http://creativecommons.org/licenses/by/2.0), which permits unrestricted use, distribution, and reproduction in any medium, provided the original work is properly cited. 
Here $J$ be any interval in $\mathbb{R}$.

Theorem 1.2. Let $\boldsymbol{x}$ and $\boldsymbol{y}$ be two $n$-tuples such that $x_{i}, y_{i} \in J, i=1, \ldots, n$; then

$$
\Lambda_{1}(f)=\sum_{i=1}^{n} f\left(y_{i}\right)-\sum_{i=1}^{n} f\left(x_{i}\right) \geq 0,
$$

holds for all continuous convex functions $f: \mathbb{R} \rightarrow \mathbb{R}$ iff $\boldsymbol{x} \prec \boldsymbol{y}$.

If $f(x)$ is a strictly convex function then equality in $(A 1)$ is valid iff $x_{[i]}=y_{[i]}, i=1, \ldots$ , n.

In 1947, Fuchs gave a weighted generalization of the well-known majorization theorem for convex functions and two sequences monotonic in the same sense [5], (see also [[3], p. 323], [[1], p. 580]).

Theorem 1.3. Let $\boldsymbol{x}$ and $\boldsymbol{y}$ be two decreasing real $n$-tuples, let $\mathbf{p}=\left(p_{1}, \ldots, p_{n}\right)$ be a real $n$-tuple such that

$$
\sum_{i=1}^{k} p_{i} x_{i} \leq \sum_{i=1}^{k} p_{i} y_{i} \text { for } k=1, \ldots, n-1 ;
$$

and

$$
\sum_{i=1}^{n} p_{i} x_{i}=\sum_{i=1}^{n} p_{i} y_{i}
$$

then, for every continuous convex function $f: J \rightarrow \mathbb{R}$, we have

$$
\Lambda_{2}(f)=\sum_{i=1}^{n} p_{i} f\left(y_{i}\right)-\sum_{i=1}^{n} p_{i} f\left(x_{i}\right) \geq 0
$$

A number of important inequalities arise from the logarithmic convexity of some functions as one can see in [1]. Logarithmic convexity plays an important role in fields of application such as reliability and survival analysis, and in economics. We also find its applications in applied mathematics as well.

Here, we give an important result from Anwar et al. [6] which is given in recent book of Marshall et al. [1].

Proposition 1.4. If $\boldsymbol{p} \in \mathbb{R}^{n}$ and $\boldsymbol{x}, \boldsymbol{y}$ are decreasing $n$-tuples such that (1) and (2) hold and $\phi_{s}:(0, \infty) \rightarrow \mathbb{R}$ defined as,

$$
\varphi_{s}(x)=\left\{\begin{array}{l}
\frac{x^{s}}{s(s-1)}, \quad s \neq 0,1 \\
-\ln x, \quad s=0 \\
x \ln x, \quad s=1
\end{array}\right.
$$

then, $\Lambda_{2}\left(\phi_{s}\right)$ is log-convex as a function of $s \in \mathbb{R}$, with $\boldsymbol{x}, \boldsymbol{y}$, and $\boldsymbol{p}$ fixed.

The following theorem is consequence of Theorem 1 in [7] (see also [[3], p. 328]):

Theorem 1.5. Let $x, y:[a, b] \rightarrow \mathbb{R}$ be increasing continuous functions and let $H:[a$, $b] \rightarrow \mathbb{R}$ be a function of bounded variation. If

$$
\int_{v}^{b} x(z) d H(z) \leq \int_{v}^{b} y(z) d H(z) \text { for every } v \in(a, b)
$$


and

$$
\int_{a}^{b} x(z) d H(z)=\int_{a}^{b} \gamma(z) d H(z)
$$

hold. Then for every continuous convex function $f$, we have

$$
\Lambda_{3}(f)=\int_{a}^{b} f(\gamma(z)) d H(z)-\int_{a}^{b} f(x(z)) d H(z) \geq 0 .
$$

Here we give another important result from Anwar et. al. [6] which is also given in [1].

Proposition 1.6. If $x, y:[0,1] \rightarrow \mathbb{R}$ are decreasing continuous functions, $H:[0,1] \rightarrow$ $\mathbb{R}$ is a function of bounded variation, and

$$
\begin{aligned}
& \int_{0}^{u} x(z) d H(z) \leq \int_{0}^{u} y(z) d H(z), \quad u \in(0,1), \\
& \int_{0}^{1} x(z) d H(z)=\int_{0}^{1} y(z) d H(z),
\end{aligned}
$$

are valid. Then

$$
\Lambda\left(\varphi_{s}\right)=\int_{0}^{1} \varphi_{s}(\gamma(z)) d H(z)-\int_{0}^{1} \varphi_{s}(x(z)) d H(z)
$$

is log-convex in $s$.

We can find variety of applications of the Propositions 1.4 and 1.6 in article [8] but we quote here an application in Statistics which is given in the book of Marshall et al. [1].

Corollary 1.7. If $W$ is positive random variable for which expectation exist and $\alpha \geq$ $\beta$, then the function

$$
\begin{gathered}
g(t)=\frac{E W^{\alpha t}-\left(E W^{\beta t}\right)\left(E W^{\alpha} / E W^{\beta}\right)^{t}}{t(t-1)}, \quad t \neq 0,1 ; \\
g(t)=\left(\log E W^{\alpha}-E \log W^{\alpha}\right)-\left(\log E W^{\beta}-E \log W^{\beta}\right), \quad t=0 ; \\
g(t)=E\left(W^{\alpha} \log W^{\alpha}\right)-\left(E W^{\alpha}\right)\left(\log E W^{\alpha}\right)-E\left(W^{\beta} \log W^{\beta}\right) \\
-\left(E W^{\beta}\right)\left(\log E W^{\beta}\right)\left(E W^{\alpha} / E W^{\beta}\right), \quad t=1
\end{gathered}
$$

is $\log$ convex.

The Propositions 1.4 and 1.6 give us log-convexity but we can find more generalized results proved by Anwar et al. [6] which give us positive semi-definite matrices and exponential convexity for positive $n$-tuples $\times$ and y. Also in [8] we find similar results for non-negative and for real $n$-tuples. But, in this article, we give much more general results than results of $[6,8]$ in new direction by using second-order divided difference.

Let $F(z)$ and $G(z)$ be two real, continuous and increasing functions for $z \geq 0$ such that $F(0)=G(0)=0$ and define 


$$
\bar{F}(z)=1-F(z), \quad \bar{G}(z)=1-G(z) \quad \text { for } \quad z \geq 0 .
$$

Definition 1.8. [[3], p. 330] $\bar{G}(z)$ is said to be majorized by $\bar{F}(z)$, in symbol, $\bar{G}(z) \prec \bar{F}(z)$, for $z \in[0, \infty)$ if

$$
\int_{0}^{s} \bar{G}(z) d z \leq \int_{0}^{s} \bar{F}(z) d z \text { for all } s>0,
$$

and

$$
\int_{0}^{\infty} \bar{G}(z) d z=\int_{0}^{\infty} \bar{F}(z) d z<\infty .
$$

The following result was obtained by Boland and Proschan [9] (see also [[3], p. 331]):

Theorem 1.9. $\bar{G}(z) \prec \bar{F}(z)$ for $z \in[0, \infty)$ holds iff

$$
\Lambda_{4}(f)=\int_{0}^{\infty} f(z) d G(z)-\int_{0}^{\infty} f(z) d F(z) \geq 0
$$

holds for all convex functions $f$, provided the integrals are finite.

Here $I$ stands for open interval in $\mathbb{R}$.

The following useful definitions are extracted from [10].

Definition 1.10. A positive function $\psi$ is $\log$-convex in the $J$-sense on $I$, if for each $x$, $y \in I$,

$$
\psi^{2}\left(\frac{x+y}{2}\right) \leq \psi(x) \psi(y)
$$

Remark 1.11. A positive function $\psi$ is log-convex in the $J$-sense on $I$ iff the relation

$$
u^{2} \psi(x)+2 u v \psi\left(\frac{x+y}{2}\right)+v^{2} \psi(y) \geq 0
$$

holds $\forall u, v \in \mathbb{R} ; x, y \in I$.

Definition 1.12. A function $\psi: I \rightarrow(0, \infty)$ is said to be a log-convex function if for every $x, y \in I$ and every $\lambda \in[0,1]$, we have

$$
\psi(\lambda x+(1-\lambda) y) \leq \psi^{\lambda}(x) \psi^{1-\lambda}(y) .
$$

The following definition is originally given by Bernstein [11] (see also [6,12-15]).

Definition 1.13. A function $\psi: I \rightarrow \mathbb{R}$ is exponentially convex on $I$ if it is continuous and

$$
\sum_{i, j=1}^{n} \xi_{i} \xi_{j} \psi\left(x_{i}+x_{j}\right) \geq 0
$$

$\forall n \in \mathbb{N}$ and all choices $\xi_{i} \in \mathrm{R} ; i=1, \ldots, n$ such that $x_{i}+x_{j} \in I ; 1 \leq i, j \leq n$.

Example 1.14. For constants $c \geq 0$ and $k \in \mathbb{R} ; x \mapsto c e^{k x}$, is an example of exponentially convex function.

The following proposition is given in [13]: 
Proposition 1.15. Let $\psi: I \rightarrow \mathbb{R}$, the following propositions are equivalent:

(i) $\psi$ is exponentially convex on $I$.

(ii) $\psi$ is continuous and

$$
\sum_{i, j=1}^{n} \xi_{i} \xi_{j} \psi\left(\frac{x_{i}+x_{j}}{2}\right) \geq 0
$$

$\forall \xi_{i} \in \mathbb{R}$ and every $x_{i} \in I ; 1 \leq i \leq n$.

Corollary 1.16. If $\psi$ is exponentially convex function on I, then the matrix

$$
\left[\psi\left(\frac{x_{i}+x_{j}}{2}\right)\right]_{i, j=1}^{n}
$$

is a positive semi-definite matrix. Particularly

$$
\operatorname{det}\left[\psi\left(\frac{x_{i}+x_{j}}{2}\right)\right]_{i, j=1}^{n} \geq 0,
$$

$\forall n \in \mathbb{N}, x_{i} \in I ; i=1, \ldots, n$.

Corollary 1.17. If $\psi: I \rightarrow(0, \infty)$ is exponentially convex function, then $\psi$ is a log-convex function.

Definition 1.18. [[3], p. 2] A function $\psi$ is convex on an interval $J \subseteq \mathbb{R}$, if

$$
\left(x_{3}-x_{2}\right) \psi\left(x_{1}\right)+\left(x_{1}-x_{3}\right) \psi\left(x_{2}\right)+\left(x_{2}-x_{1}\right) \psi\left(x_{3}\right) \geq 0
$$

holds for every $x_{1}<x_{2}<x_{3} ; x_{1}, x_{2}, x_{3} \in J$.

This article has been divided into three sections. In Section 1, we have given introduction and preliminaries. In Section 2, we investigate the log-convexity and exponential convexity for majorization type results for discrete as well as continuous cases by using classes of continuous functions in linear functionals defined in $(A 1), \ldots,(A 4)$. We also construct positive semi-definite matrices for majorization type results. In Section 3, we will vary on choice of a family of functions in order to construct different examples of exponentially convex functions and construct some means. We also prove the monotonic property.

\section{Main results}

Let $f$ be a real-valued function defined on $[a, b]$, a second-order divided difference of $f$ at distinct points $z_{0}, z_{1}, z_{2} \in[a, b]$ is defined (as in [[3], p. 14]) recursively by

$$
\begin{aligned}
& {\left[z_{i} ; f\right]=f\left(z_{i}\right), \quad \text { for } \quad i=0,1,2 ;} \\
& {\left[z_{i}, z_{i+1} ; f\right]=\frac{f\left(z_{i+1}\right)-f\left(z_{i}\right)}{z_{i+1}-z_{i}}, \text { for } i=0,1 ;}
\end{aligned}
$$

and

$$
\left[z_{0}, z_{1}, z_{2} ; f\right]=\frac{\left[z_{1}, z_{2} ; f\right]-\left[z_{0}, z_{1} ; f\right]}{z_{2}-z_{0}} .
$$

The value $\left[z_{0}, z_{1}, z_{2} ; f\right]$ is independent of the order of the points $z_{0}, z_{1}$, and $z_{2}$. By taking limits this definition may be extended to include the cases in which any two or all 
three points coincide as follows: $\forall z_{0}, z_{1}, z_{2} \in[a, b]$

$$
\left[z_{0}, z_{0}, z_{2} ; f\right]=\lim _{z_{1} \rightarrow z_{0}}\left[z_{0}, z_{1}, z_{2} ; f\right]=\frac{f\left(z_{2}\right)-f\left(z_{0}\right)-f^{\prime}\left(z_{0}\right)\left(z_{2}-z_{0}\right)}{\left(z_{2}-z_{0}\right)^{2}}, z_{2} \neq z_{0}
$$

given that $f$ exists on $[\mathrm{a}, \mathrm{b}]$ and

$$
\left[z_{0}, z_{0}, z_{0} ; f\right]=\lim _{z_{i} \rightarrow z_{0}}\left[z_{0}, z_{1}, z_{2} ; f\right]=\frac{f^{\prime \prime}\left(z_{0}\right)}{2} \text { for } i=1,2
$$

provided that $f^{\prime \prime}$ exists on $[\mathrm{a}, \mathrm{b}]$.

Let $K_{2}[a, b]$ be the class of all functions from $C[a, b]$ that are convex. So, $f \in K_{2}[a$, $b]$ if $\left[z_{0}, z_{1}, z_{2} ; f\right] \geq 0$; for any three points $z_{0}, z_{1}, z_{2} \in[a, b]$.

Let us define some classes to be used in the following theorem as follows:

For any open interval $I \subseteq \mathbb{R}$, and $[a, b] \subseteq D(f)$ where $D(f)$ stands for domain of $f$, we have

$D_{1}=\left\{f_{t}: t \in I\right\}$ be a class of functions from $C[a, b]$ such that the function $t \mapsto\left[z_{0}, z_{1}\right.$, $\left.z_{2} ; f_{t}\right]$ is log-convex in $J$-sense on $I$ for any three distinct points $z_{0}, z_{1}, z_{2} \in[a, b]$.

$D_{2}=\left\{f_{t}: t \in I\right\}$ be a class of differentiable functions such that the function $t \mapsto\left[z_{0}\right.$, $\left.z_{0}, z_{2} ; f_{t}\right]$ is log-convex in $J$-sense on $I$ for any two distinct points $z_{0}, z_{2} \in[a, b]$.

$D_{3}=\left\{f_{t}: t \in I\right\}$ be a class of twice differentiable functions such that the function $t \mapsto$ $\left[z_{0}, z_{0}, z_{0} ; f_{t}\right]$ is log-convex in $J$-sense on $I$ for any point $z_{0} \in[a, b]$.

Theorem 2.1. Let $\Lambda_{k}$ be linear functionals for $k=1, \ldots, 4$ as defined in $(A 1), \ldots$, (A4). Let $I$ be any open interval in $\mathbb{R}$ and $f_{t} \in D_{j}, j=1,2,3, t \in I$, we also assume that for $\Lambda_{1}\left(f_{t}\right), x_{[i]} \neq y_{[i]}, i=1, \ldots, n$; and $\Lambda_{k}\left(f_{t}\right)$ are positive for $k=2,3,4$, then the following statements are valid for $\Lambda_{k} ; k=1, \ldots, 4$ :

(a) The function $t \mapsto \Lambda_{k}\left(f_{t}\right)$ is log-convex in J-sense on $I$.

(b) If the function $t \mapsto \Lambda_{k}\left(f_{t}\right)$ is continuous on $I$, then the function $t \mapsto \Lambda_{k}\left(f_{t}\right)$ is logconvex on I. Also, the following inequality holds for $r<s<t ; r, s, t \in I$

$$
\left[\Lambda_{k}\left(f_{s}\right)\right]^{t-r} \leq\left[\Lambda_{k}\left(f_{r}\right)\right]^{t-s}\left[\Lambda_{k}\left(f_{t}\right)\right]^{s-r}
$$

which is called Lyapunov's inequality.

(c) If the function $t \mapsto \Lambda_{k}\left(f_{t}\right)$ is derivable on $I$, then $\forall s \leq u$ and $t \leq v$; $s, t, u, v \in I$; we have

$$
\mu_{s, t}\left(\Lambda_{k}, D_{j}\right) \leq \mu_{u, v}\left(\Lambda_{k}, D_{j}\right) \quad \text { for } j=1,2,3 ;
$$

where

$$
\mu_{s, t}\left(\Lambda_{k}, D_{j}\right)=\left\{\begin{array}{l}
\left(\frac{\Lambda_{k}\left(f_{s}\right)}{\Lambda_{k}\left(f_{t}\right)}\right)^{\frac{1}{s-t}}, \quad s \neq t \\
\exp \left(\frac{\frac{d}{d_{s}} \Lambda_{k}\left(f_{s}\right)}{\Lambda_{k}\left(f_{s}\right)}\right), s=t
\end{array}\right.
$$

Proof. (a) Since, the function $t \mapsto\left[z_{0}, z_{1}, z_{2} ; f_{t}\right]$ is log-convex in $J$-sense on $I$ which implies that $\left[z_{0}, z_{1}, z_{2} ; f_{t}\right]>0$. So, $f_{t}$ is strictly convex and $x_{[i]} \neq y_{[i]}$ for $i=1, \ldots, n$; which shows that by using Theorem $1.2, \Lambda_{1}\left(f_{t}\right)$ is positive and also by our assumptions that $\Lambda_{k}\left(f_{t}\right)$ for $k=2,3,4$ are positive. 
Now for any fixed $p, q \in \mathbb{R}$ and $s, t \in I$, we define the following function

$$
g(z)=p^{2} f_{s}(z)+2 p q f_{\frac{s+t}{2}}(z)+q^{2} f_{t}(z)
$$

by using Remark 1.11, the log-convexity in $J$-sense of the function $t \mapsto\left[z_{0}, z_{1}, z_{2} ; f_{t}\right]$ gives us the positive definiteness of the quadratic form

$$
\left[z_{0}, z_{1}, z_{2} ; g\right]=p^{2}\left[z_{0}, z_{1}, z_{2} ; f_{s}\right]+2 p q\left[z_{0}, z_{1}, z_{2} ; f_{\frac{s+t}{2}}\right]+q^{2}\left[z_{0}, z_{1}, z_{2} ; f_{t}\right] \geq 0
$$

which implies $g$ is convex function and clearly $g$ is continuous therefore $g \in K_{2}[a, b]$ and we have $\Lambda_{k}(g) \geq 0$ for $k=1, \ldots, 4$. Hence we have

$$
p^{2} \Lambda_{k}\left(f_{s}\right)+2 p q \Lambda_{k}\left(f_{\frac{s+t}{2}}\right)+q^{2} \Lambda_{k}\left(f_{t}\right) \geq 0
$$

so, the function $t \mapsto \Lambda_{k}\left(f_{t}\right)$ is log-convex in $J$-sense on $I$ for $k=1, \ldots, 4$.

(b) If the function $t \mapsto \Lambda_{k}\left(f_{t}\right)$ is in addition continuous, then from (a) we conclude that the function is log-convex on $I$.

As the function $t \mapsto \Lambda_{k}\left(f_{t}\right)$ is log-convex, i.e., $\ln \Lambda_{k}\left(f_{t}\right)$ is convex, so by using Definition 1.18 , we have

$$
\ln \left[\Lambda_{k}\left(f_{s}\right)\right]^{t-r} \leq \ln \left[\Lambda_{k}\left(f_{r}\right)\right]^{t-s}+\ln \left[\Lambda_{k}\left(f_{t}\right)\right]^{s-r}, k=1, \ldots, 4 ;
$$

which gives us (4).

(c) By definition of convex function $\varphi$, we have the following inequality [3, p. 2]

$$
\frac{\phi(s)-\phi(t)}{s-t} \leq \frac{\phi(u)-\phi(v)}{u-v}
$$

$\forall s, t, u, v \in I$ with $s \leq u, t \leq v, s \neq t, u \neq v$.

Since by (b), $\Lambda_{k}\left(f_{t}\right)$ is log-convex for $k=1, \ldots, 4$ so in (6) we set $\varphi(x)=\ln \Lambda_{k}\left(f_{x}\right)$, to get

$$
\frac{\ln \Lambda_{k}\left(f_{s}\right)-\ln \Lambda_{k}\left(f_{t}\right)}{s-t} \leq \frac{\ln \Lambda_{k}\left(f_{u}\right)-\ln \Lambda_{k}\left(f_{v}\right)}{u-v} ; k=1, \ldots, 4
$$

which gives us

$$
\mu_{s, t}\left(\Lambda_{k}, D_{j}\right) \leq \mu_{u, v}\left(\Lambda_{k}, D_{j}\right) \text { for } j=1,2,3 \text { and } k=1, \ldots, 4 \text {. }
$$

If $s=t \leq u$ we apply the limit $\lim _{t \rightarrow s}$ to (7) concluding

$$
\mu_{s, s}\left(\Lambda_{k}, D_{j}\right) \leq \mu_{u, v}\left(\Lambda_{k}, D_{j}\right) \text { for } j=1,2,3 \text { and } k=1, \ldots, 4 \text {. }
$$

Other possible cases can be treated in the same way. $\square$

Now, we give two important remarks and one useful corollary from [10], which we will use in some examples in third section.

Remark 2.2. For $\mu_{s, t}\left(\Lambda_{k}, D\right)$ defined with (5) we will refer as mean if

$$
a \leq \mu_{s, t}\left(\Lambda_{k}, D\right) \leq b
$$

for $s, t \in I$ and $k=1, \ldots, 4$.

We can find mean value theorems of Cauchy's type for $\Lambda_{k}\left(f_{t}\right)$ for $k=1, \ldots, 4$ as in $[6,8]$. We can also apply second mean value theorem of Cauchy's type for $\Lambda_{k}\left(f_{t}\right), k=1$, 
$\ldots, 4$ in order to get criteria for recognition of means as in [10]. This theorem gives us the following corollary:

Corollary 2.3. Let $a, b \in \mathbb{R}$ and $\Lambda_{k}$ be linear functionals for $k=1, \ldots, 4$ as defined in (A1), .., (A4). Let $D=\left\{f_{t}: t \in I\right\}$ be a family of functions in $C^{2}[a, b]$. If

$$
a \leq\left(\frac{\frac{d^{2} f_{s}}{d x^{2}}}{\frac{d^{2} f_{t}}{d x^{2}}}\right)^{\frac{1}{s-t}}(\xi) \leq b,
$$

for $\xi \in[a, b] ; s, t \in I$; then $\mu_{s, t}\left(\Lambda_{k}, D\right)$ is a mean for $k=1, \ldots, 4$.

Remark 2.4. In some examples, we will have very simple recognition of means:

$$
\left(\frac{\frac{d^{2} f_{s}}{d x^{2}}}{\frac{d^{2} f_{t}}{d x^{2}}}\right)^{\frac{1}{s-t}}(\xi)=\xi, \quad \xi \in[a, b], s \neq t .
$$

Let us define some new classes to be used in next theorem as follows:

For any open interval $I \subseteq \mathrm{R}$, and $[a, b] \subseteq D(f)$ where $D(f)$ stands for domain of $f$, we have

$\tilde{D}_{1}=\left\{f_{t}: t \in I\right\}$ be a class of functions from $C[a, b]$ such that the function $t \mapsto\left[z_{0}, z_{1}\right.$, $\left.z_{2} ; f_{t}\right]$ is exponentially convex on $I$ for any three distinct points $z_{0}, z_{1}, z_{2} \in[a, b]$.

$\tilde{D}_{2}=\left\{f_{t}: t \in I\right\}$ be a class of differentiable functions such that the function $t \mapsto\left[z_{0}\right.$, $\left.z_{0}, z_{2} ; f_{t}\right]$ is exponentially convex on $I$ for any two distinct points $z_{0}, z_{2} \in[a, b]$.

$\tilde{D}_{3}=\left\{f_{t}: t \in I\right\}$ be a class of twice differentiable functions such that the function $t \mapsto$ $\left[z_{0}, z_{0}, z_{0} ; f_{t}\right]$ is exponentially convex on $I$ for any point $z_{0} \in[a, b]$.

Theorem 2.5. Let $\Lambda_{k}$ be linear functionals for $k=1, \ldots, 4$ as defined in $(A 1), \ldots$, (A4) and let $I$ be any open interval in $\mathbb{R}$, then for $f_{t} \in \tilde{D}_{j}, j=1,2,3$ and $t \in I$, the following statements are valid for $\Lambda_{k} ; k=1, \ldots, 4$ :

(a) For every $n \in \mathbb{N}$ and $t_{1}, \ldots, t_{n} \in I$, the matrix $\left[\Lambda_{k}\left(f_{\frac{t_{i}+t_{j}}{2}}\right)\right]_{i, j=1}^{n}$ is a positive semi-definite. Particularly

$$
\operatorname{det}\left[\Lambda_{k}\left(\frac{f_{t}+t_{j}}{2}\right)\right]_{i, j=1}^{n} \geq 0 .
$$

(b) If the function $t \mapsto \Lambda_{k}\left(f_{t}\right)$ is continuous on $I$, then it is exponentially convex on I.

(c) If $\Lambda_{k}\left(f_{t}\right)$ be positive for $k=2,3,4$ and for $\Lambda_{1}\left(f_{t}\right)$ we assume that $f_{t}$ is strictly convex with condition $x_{[i]} \neq y_{[i]}$ for $i=1, \ldots, n$ and the function $t \mapsto \Lambda_{k}\left(f_{t}\right)$ is derivable on I then $\forall s \leq u$ and $t \leq v$, we have

$$
\mu_{s, t}\left(\Lambda_{k}, \tilde{D}_{j}\right) \leq \mu_{u, v}\left(\Lambda_{k}, \tilde{D}_{j}\right) \quad \text { for } j=1,2,3 ;
$$

where $\mu_{s, t}\left(\Lambda_{k}, \tilde{D}_{j}\right)$ is as defined in (5). 
Proof. (a) We consider the function

$$
h_{l}(z)=\sum_{i, j=1}^{l} u_{i} u_{j} \frac{f_{t_{i}+t_{j}}}{2}(z)
$$

for $l=1, \ldots, n$ and $f_{t} \in \tilde{D}_{1}$, where $z \in[a, b], u_{i} \in \mathbb{R}$ and $t_{i} \in I$.

Since $\left[z_{0}, z_{1}, z_{2} ; \frac{f_{t_{i}+t_{j}}}{2}\right]$ is exponentially convex function for $z_{0}, z_{1}, z_{2} \in[a, b]$, so

$$
\left[z_{0}, z_{1}, z_{2} ; h_{l}\right]=\sum_{i, j}^{l} u_{i} u_{j}\left[z_{0}, z_{1}, z_{2} ; \frac{f_{t_{i}+t_{j}}}{2}\right] \geq 0
$$

which shows that $h_{l}$ is a convex function for $l=1, \ldots, n$ and $z_{0}, z_{1}, z_{2} \in[a, b]$. Now by using Theorem 1.2,

$$
\sum_{m=1}^{n} h_{l}\left(y_{m}\right)-\sum_{m=1}^{n} h_{l}\left(x_{m}\right) \geq 0, \quad \text { for } \quad l=1, \ldots, n
$$

we get

$$
\sum_{m=1}^{n}\left(\sum_{i, j=1}^{l} u_{i} u_{j} f_{\frac{t_{i}+t_{j}}{2}}\left(y_{m}\right)\right)-\sum_{m=1}^{n}\left(\sum_{i, j=1}^{l} u_{i} u_{j} \frac{f_{t_{i}+t_{j}}}{2}\left(x_{m}\right)\right) \geq 0,
$$

for $l=1, \ldots, n$ or

$$
\sum_{i, j=1}^{l} u_{i} u_{j} \Lambda_{k}\left(\frac{f_{t_{i}+t_{j}}}{2}\right) \geq 0, \quad \text { for } \quad l=1, \ldots, n \text { and } k=1, \ldots, 4
$$

which shows that the matrix $\left[\Lambda_{k}\left(\frac{f_{i}+t_{j}}{2}\right)\right]_{i, j=1}^{n}$ is a positive semi-definite, and by using Sylvester criteria we get (8).

(b) By our assumption $\Lambda_{k}\left(f_{t}\right)$ is continuous for $t \in I$ and $k=1, \ldots, 4$; then by using Proposition 1.15, we get exponential convexity of the function $t \mapsto \Lambda_{k}\left(f_{t}\right)$ on $I$ for $k=1$, ..., 4 .

(c) As $\Lambda_{k}\left(f_{t}\right)$ is positive and the function $t \mapsto \Lambda_{k}\left(f_{t}\right)$ is derivable for $k=1, \ldots, 4$ by our assumption, hence the result follows directly by using (c) part of Theorem 2.1.

\section{Examples}

In this section, we will vary on choice of a family $D=\left\{f_{t}: t \in I\right\}$ for any open $I \subset \mathbb{R}$ in order to construct different examples of exponentially convex functions and construct some means.

Let us consider some examples:

Example 3.1. Let

$$
D=\left\{\varphi_{s}:(0, \infty) \rightarrow \mathbb{R}: s \in \mathbb{R}\right\}
$$

be a family of functions defined in (3). Since $\frac{d^{2}}{d x^{2}} \varphi_{s}(x)=x^{s-2}=e^{(s-2) \ln x}$ for $x>0$ so $s \mapsto \frac{d^{2}}{d x^{2}} \varphi_{s}$ is exponentially convex function with respect to $s$ by Example 1.14. So, $\Lambda_{k}$ $\left(\phi_{s}\right), k=1, \ldots, 4$ are exponentially convex by Theorem 2.5 and by Corollary 1.17 
every positive exponentially convex function is log-convex. So, if we choose class of functions given in (3) in Theorem 2.5 then for $\Lambda_{1}$ we get Theorems 2.4 and 2.5 of [6] and similarly for $\Lambda_{k}, k=2,3$; the Theorems 2.6, 2.7, 4.4, 4.5, 4.11, and 4.12 of [6] all become special cases of Theorem 2.5 of this article. We can also obtain similar results for $\Lambda_{4}$ as in [6].

Further, in this choice of family $D$ we have

$$
\left(\frac{\frac{d^{2} \varphi_{s}}{d x^{2}}}{\frac{d^{2} \varphi_{t}}{d x^{2}}}\right)^{\frac{1}{s-t}}(\xi)=\xi, \quad \xi \in[a, b], s \neq t, \text { where } a, b \in(0, \infty) .
$$

So, using Remark 2.4 we have an important conclusion that $\mu_{s, t}\left(\Lambda_{k}, D\right)$ is in fact mean for $k=1, \ldots, 4$. We can extend these means in other cases as given in [6].

Example 3.2. Let

$$
\bar{D}=\left\{\bar{\varphi}_{s}:[0, \infty) \rightarrow \mathbb{R}: s \in(0, \infty)\right\}
$$

be a family of functions defined as

$$
\bar{\varphi}_{s}(x)=\left\{\begin{array}{l}
\frac{x^{s}}{s(s-1)}, s \neq 1 \\
x \ln x, s=1
\end{array}\right.
$$

here, we use the convention $0 \ln 0=0$.

Since, $\frac{d^{2}}{d x^{2}} \bar{\varphi}_{s}(x)=x^{s-2}=e^{(s-2) \ln x}$ for $x>0$ so by same argument given in previous example we conclude that $\Lambda_{k}\left(\bar{\varphi}_{s}\right), k=1, \ldots, 4$ are exponentially convex functions with respect to $s$. So, if we choose class of functions given in (9) in Theorem 2.5 then we get Theorems 18 and 19 of [8] for $\Lambda_{1}$ and similarly for $\Lambda_{k}, k=2,3,4$; Theorems $20,26,27,28$, and 29 of [8] become special cases of Theorem 2.5 of this article.

Further, in this choice of family $\bar{D}$ we have

$$
\left(\frac{\frac{d^{2} \bar{\varphi}_{s}}{d x^{2}}}{\frac{d^{2} \bar{\varphi}_{t}}{d x^{2}}}\right)^{\frac{1}{s-t}}(\xi)=\xi, \quad \xi \in[a, b], s \neq t, \text { where } a, b \in(0, \infty) .
$$

So, using Remark 2.4 we have an important conclusion that $\mu_{s, t}\left(\Lambda_{k}, \bar{D}\right)$ is in fact mean for $k=1, \ldots, 4$. We can extend these means in other cases as given in [8]. All the means $\mu_{s, t}\left(\Lambda_{k}, \bar{D}\right)$ are calculated for two parameters $s$ and $t$, now we move towards three parameters namely $s, t, r$. For $r>0$ by substituting $x_{i}=x_{i}^{r}, y_{i}=y_{i}^{r}, t=\frac{t}{r}, s=\frac{s}{r}$ in $\mu_{s, t}\left(\Lambda_{k}, \bar{D}\right)$, we get similar results as given in [8].

Example 3.3. Let

$$
\Psi=\left\{\psi_{s}: \mathbb{R} \rightarrow[0, \infty): s \in \mathbb{R}\right\}
$$

defined as

$$
\psi_{s}(x)=\left\{\begin{array}{l}
\frac{1}{s^{2}} e^{s x}, s \neq 0 \\
\frac{1}{2} x^{2}, s=0
\end{array}\right.
$$

Since, $\frac{d^{2}}{d x^{2}} \psi_{s}(x)=e^{s x}$ for $x \in \mathbb{R}$ so by same argument given in Example 3.1 we conclude that $\Lambda_{k}\left(\psi_{s}\right), k=1, \ldots, 4$ are exponentially convex functions with respect to $s$. 
So, if we choose this class of function in Theorem 2.5 then for $\Lambda_{1}$ we get Theorem 32 of [8] and similarly for $\Lambda_{k}, k=2,3,4$; the Theorems 34, 40, 41, and 42 of [8] all become special cases of Theorem 2.5 of this article.

Monotonicity: if $s, t, u, v \in \mathbb{R}$ such that $s \leq u, t \leq v$, then by using Theorem 2.5 we have

$$
\mu_{s, t}\left(\Lambda_{k}, \Psi\right) \leq \mu_{u, v}\left(\Lambda_{k}, \Psi\right) \text { for } k=1, \ldots, 4
$$

We observe here that $\left(\frac{\frac{d^{2} \psi_{s}}{\frac{d^{2}}{d^{2} \psi_{t}}}}{d x^{2}}\right)^{\frac{1}{s-t}}(\ln \xi)=\xi$ is a mean for $\xi \in[a, b]$ where $a, b \in \mathbb{R}$.

We also note that after the substitution $\left(x_{i}, y_{i}\right) \rightarrow\left(\ln x_{i}, \ln y_{i}\right) ; i=1, \ldots, n$ in $\Lambda_{k}, k$ $=1,2$ in Theorem 2.5 we will get the Corollaries 36 and 38 of [8]. We can also obtain similar results for $\Lambda_{3}$ and $\Lambda_{4}$ by this substitution in Theorem 2.5 .

Example 3.4. Let

$$
\Theta=\left\{\theta_{s}:(0, \infty) \rightarrow(0, \infty): s \in(0, \infty)\right\}
$$

be family of functions defined with

$$
\theta_{s}(x)=\frac{e^{-x \sqrt{s}}}{s}
$$

Since $\frac{d^{2}}{d x^{2}} \theta_{s}(x)=e^{-x \sqrt{s}}$, for $x>0$ so by same argument given in Example 3.1 we conclude that $\Lambda_{k}\left(\theta_{s}\right), k=1, \ldots, 4$ are exponentially convex functions with respect to $s$.

For this family of functions we have the following possible cases of $\mu_{s, t}$ :

$$
\begin{aligned}
& \mu_{s, t}\left(\Lambda_{1}, \Theta\right)=\left\{\begin{array}{l}
\left(\frac{\Lambda_{1}\left(\theta_{s}\right)}{\Lambda_{1}\left(\theta_{t}\right)}\right)^{\frac{1}{s-t}}, \quad s \neq t \\
\exp \left(\frac{\sum_{i=1}^{n} y_{i} e^{-y_{i} \sqrt{s}}-\sum_{i=1}^{n} x_{i} e^{-x_{i} \sqrt{s}}}{2 \sqrt{s}\left(\sum_{i=1}^{n} e^{-x_{i} \sqrt{s}}-\sum_{i=1}^{n} e^{-\gamma_{i} \sqrt{s}}\right)}-\frac{1}{s}\right), \quad s=t,
\end{array}\right. \\
& \mu_{s, t}\left(\Lambda_{2}, \Theta\right)=\left\{\begin{array}{l}
\left(\frac{\Lambda_{2}\left(\theta_{s}\right)}{\Lambda_{2}\left(\theta_{t}\right)}\right)^{\frac{1}{s-t}}, \quad s \neq t ; \\
\exp \left(\frac{\sum_{i=1}^{n} p_{i} \gamma_{i} e^{-y_{i} \sqrt{s}}-\sum_{i=1}^{n} p_{i} x_{i} e^{-x_{i} \sqrt{s}}}{2 \sqrt{s}\left(\sum_{i=1}^{n} p_{i} e^{-x_{i} \sqrt{s}}-\sum_{i=1}^{n} p_{i} e^{-y_{i} \sqrt{s}}\right)}-\frac{1}{s}\right), \quad s=t,
\end{array}\right. \\
& \mu_{s, t}\left(\Lambda_{3}, \Theta\right)=\left\{\begin{array}{l}
\left(\frac{\Lambda_{3}\left(\theta_{s}\right)}{\Lambda_{3}\left(\theta_{t}\right)}\right)^{\frac{1}{s-t}, \quad s \neq t ;} \\
\exp \left(\frac{\int_{a}^{b} \gamma(z) e^{-\gamma(z) \sqrt{s}} d H(z)-\int_{a}^{b} x(z) e^{-x(z) \sqrt{s}} d H(z)}{2 \sqrt{s}\left(\int_{a}^{b} e^{-x(z) \sqrt{s}} d H(z)-\int_{a}^{b} e^{-\gamma(z) \sqrt{s}} d H(z)\right)}-\frac{1}{s}\right), \quad s=t,
\end{array}\right. \\
& \mu_{s, t}\left(\Lambda_{4}, \Theta\right)=\left\{\begin{array}{l}
\left(\frac{\Lambda_{4}\left(\theta_{s}\right)}{\Lambda_{4}\left(\theta_{t}\right)}\right)^{\frac{1}{s-t}}, \quad s \neq t ; \\
\exp \left(\frac{\int_{0}^{\infty} z e^{-z \sqrt{s}} d G(z)-\int_{0}^{\infty} z e^{-z \sqrt{s}} d F(z)}{2 \sqrt{s}\left(\int_{0}^{\infty} e^{-z \sqrt{s}} d F(z)-\int_{0}^{\infty} e^{-z \sqrt{s}} d G(z)\right)}-\frac{1}{s}\right), \quad s=t .
\end{array}\right.
\end{aligned}
$$

Monotonicity: if $s, t, u, v \in(0, \mathbb{R})$ such that $s \leq u, t \leq v$, then by using Theorem 2.5 we have 


$$
\mu_{s, t}\left(\Lambda_{k}, \Theta\right) \leq \mu_{u, v}\left(\Lambda_{k}, \Theta\right) \quad \text { for } k=1, \ldots, 4
$$

Example 3.5. Let

$$
\Phi=\left\{\phi_{s}:(0, \infty) \rightarrow(0, \infty): s \in(0, \infty)\right\}
$$

be family of functions defined with

$$
\phi_{s}(x)=\left\{\begin{array}{cc}
\frac{s^{-x}}{(\ln s)^{2}}, & s \neq 1 \\
\frac{x^{2}}{2}, & s=1 .
\end{array}\right.
$$

Since $\frac{d^{2}}{d x^{2}} \phi_{s}(x)=s^{-x}=e^{-x l n s}>0$, for $x>0$, so by same argument given in Example 3.1 we conclude that $\Lambda_{k}\left(\varphi_{s}\right), k=1, \ldots, 4$ are exponentially convex functions with respect to $s$.

For this family of function we have the following possible cases of $\mu_{s, t}$ :

$$
\begin{aligned}
& \mu_{s, t}\left(\Lambda_{1}, \Phi\right)=\left\{\begin{array}{l}
\left(\frac{\Lambda_{1}\left(\phi_{s}\right)}{\Lambda_{1}\left(\phi_{t}\right)}\right)^{\frac{1}{s-t}}, \quad s \neq t \\
\exp \left(\frac{\sum_{i=1}^{n} y_{i}-y_{i}-\sum_{i=1}^{n} x_{i} s^{-x_{i}}}{s\left(\sum_{i=1}^{n} s^{-x_{i}}-\sum_{i=1}^{i} s^{-\gamma_{i}}\right)}-\frac{2}{s \ln s}\right), \quad s=t \neq 1 \\
\exp \left(\frac{1}{3} \frac{\sum_{i=1}^{n} y_{i}-\sum_{i=1}^{n} x_{i}^{3}}{\sum_{i=1}^{n} x_{i}^{2}-\sum_{i=1}^{n} y_{i}^{2}}\right), \quad s=t=1
\end{array}\right. \\
& \mu_{s, t}\left(\Lambda_{2}, \Phi\right)=\left\{\begin{array}{l}
\left(\frac{\Lambda_{2}\left(\phi_{s}\right)}{\Lambda_{2}\left(\phi_{t}\right)}\right)^{\frac{1}{s-t}}, \quad s \neq t \\
\exp \left(\frac{\sum_{i=1}^{n} p_{i} y_{i} s^{-y_{i}}-\sum_{i=1}^{n} p_{i} x_{i} s^{-x_{i}}}{s\left(\sum_{i=1}^{n} p_{i} s^{-x_{i}}-\sum_{i=1}^{n} p_{i} s^{-\gamma_{i}}\right)}-\frac{2}{s \ln s}\right), \quad s=t \neq 1 \\
\exp \left(\frac{1}{3} \frac{\sum_{i=1}^{n} p_{i} \gamma_{i}^{3}-\sum_{i=1}^{n} p_{i} x_{i}^{3}}{\sum_{i=1}^{n} p_{i} x_{i}^{2}-\sum_{i=1}^{n} p_{i} y_{i}^{2}}\right), \quad s=t=1,
\end{array}\right. \\
& \mu_{s, t}\left(\Lambda_{3}, \Phi\right)=\left\{\begin{array}{l}
\left(\frac{\Lambda_{3}\left(\phi_{s}\right)}{\Lambda_{3}\left(\phi_{t}\right)}\right)^{\frac{1}{s-t}}, \quad s \neq t \\
\exp \left(\frac{\int_{a}^{b} y(z) s^{-\gamma(z)} d H(z)-\int_{a}^{b} x(z) s^{-x(z)} d H(z)}{s\left(\int_{a}^{b} s^{-x(z)} d H(z)-\int_{a}^{b} s^{-\gamma(z)} d H(z)\right)}-\frac{2}{s \ln s}\right), \quad s=t \neq 1 \\
\exp \left(\frac{1}{3} \frac{\int^{b} y^{3}(z) d H(z)-\int_{a}^{b} x^{3}(z) d H(z)}{\int_{a}^{b} x^{2}(z) d H(z)-\int_{a}^{b} \gamma^{2}(z) d H(z)}\right), \quad s=t=1,
\end{array}\right. \\
& \mu_{s, t}\left(\Lambda_{4}, \Phi\right)=\left\{\begin{array}{l}
\left(\frac{\Lambda_{4}\left(\phi_{s}\right)}{\Lambda_{4}\left(\phi_{t}\right)}\right)^{\frac{1}{s-t}}, \quad s \neq t ; \\
\exp \left(\frac{\int_{0}^{\infty} z^{-z} d G(z)-\int_{0}^{\infty} z s^{-z} d F(z)}{s\left(\int_{0}^{\infty} s^{-z} d F(z)-\int_{0}^{\infty} s^{-z} d G(z)\right)}-\frac{2}{s \ln s}\right), \quad s=t \neq 1 \\
\exp \left(\frac{1}{3} \frac{\int_{0}^{\infty} z^{3} d G(z)-\int_{0}^{\infty} z^{3} d H(z)}{\int_{0}^{\infty} z^{2} d F(z)-\int_{0}^{\infty} z^{2} d G(z)}\right), \quad s=t=1 .
\end{array}\right.
\end{aligned}
$$

Monotonicity: if $s, t, u, v \in(0, \infty)$ such that $s \leq u, t \leq v$, then by using Theorem 2.5 we have

$$
\mu_{s, t}\left(\Lambda_{k}, \Phi\right) \leq \mu_{u, v}\left(\Lambda_{k}, \Phi\right) \quad \text { for } k=1, \ldots, 4
$$


Acknowledgements

This research study was funded by Higher Education Commission Pakistan. The research of J. Pečarić was supported by the Croatian Ministry of Science, Education and Sports under the Research Grants 117-1170889-0888.

\section{Author details}

'Department of Mathematics, University of Karachi, University Road, Karachi, Pakistan ${ }^{2}$ Abdus Salam School of Mathematical Sciences, GC University, Lahore, Pakistan ${ }^{3}$ Department of Mathematics, GC University, Faisalabad, Pakistan ${ }^{4}$ Faculty of Textile Technology, University of Zagreb, Zagreb, Croatia

\section{Authors' contributions}

JP made the the main contribution in conceiving the presented research. JP, NL and ARK worked jointly on each section while ARK drafted the manuscript. All authors read and approved the final manuscript.

\section{Competing interests}

The authors declare that they have no competing interests.

Received: 22 March 2011 Accepted: 8 May 2012 Published: 8 May 2012

\section{References}

1. Marshall, AW, Olkin, l, Arnold, BC: Theory of Majorization and its Applications. Springer, New York, 2 (2011)

2. Hardy, GH, Littlewood, JE, Pólya, G: Inequalities. Cambridge University press, England, 2 (1952)

3. Pečarić, J, Proschan, F, Tong, YL: Convex Functions, Partial Orderings and Statistical Applications. Academic Press, New York (1992)

4. Mitrinović, DS: Analytic Inequalities (in co-operation with Vasić, PM). Springer Verlag, New York. (1970)

5. Fuchs, L: A new proof of an inequality of Hardy-Littlewood-Polya. Mat Tidsskr. B, 53-54 (1947)

6. Anwar, M, Latif, N, Pečarić, J: Positive semi-definite matrices, exponential convexity for majorization and related Cauchy means. Journal of Inequalities and Applications2010. Article ID 728251, 19 pages, doi:10.1155/2010/728251

7. Pečarić, J: On some inequalities for functions with nondecreasing increments. J Math Anal Appl. 98, 188-197 (1984). doi:10.1016/0022-247X(84)90287-7

8. Latif, N, Pečarić, J: Positive semi-definite matrices, exponential convexity for multiplicative Majorization and related means of Cauchy's type. Revue d'Aanlyse Numérique et de Théorie de l'Approximation (ANTA)36(1). volume 2010, Tome, $N^{\circ} 1$, pages 19

9. Boland, PJ, Proschan, F: An integral inequality with applications to order statistics. In: Basu, AP (eds.) Reliability and Quality Control. pp. 107-116. North Holland, Amsterdam (1986)

10. Jekšetić, J, Pečarić, J: Exponential Convexity Method. Journal of Convex Analysis. (in press)

11. Bernstien, SN: Sur les fonctions absolument monotones. Acta Math. 52, 1-66 (1929). doi:10.1007/BF02592679

12. Akhiezer, NI: The Classical Moment Problem and Some Related Questions in Analysis. Oliver and Boyd, Edinburgh (1965)

13. Anwar, M, Jekšetić, J, Pečarić, J, Rehman, Au: Exponential convexity, positive semi-definite matrices and fundamental inequalities. J Math Inequal 2009, 19 (2009). Article ID jmi-0376

14. Mitrinović, DS, Pečarić, JE: On Some inequalities for monotonic functions. Boll Unione Mat Ital. 5-13(7):407-416 (1991)

15. Mitrinović, DS, Pečarić, J, Fink, AM: Classical and New Inequalities in Analysis. Kluwer Academic Publishers, The Netherlands. (1993)

doi:10.1186/1029-242X-2012-105

Cite this article as: Khan et al.: Exponential convexity for majorization. Journal of Inequalities and Applications 2012 2012:105.

\section{Submit your manuscript to a SpringerOpen ${ }^{\odot}$ journal and benefit from:}

- Convenient online submission

- Rigorous peer review

- Immediate publication on acceptance

- Open access: articles freely available online

- High visibility within the field

- Retaining the copyright to your article

Submit your next manuscript at $\boldsymbol{s p r i n g e r o p e n . c o m ~}$ 